\title{
DIPOLAR CONTRIBUTIONS TO MAGNETOELASTIC ENERGY OF CRYSTAL GRAINS WITH INTERFACES
}

\author{
T. SzUmiata ${ }^{a}$, R. ŻUBEREK ${ }^{b}$, H. SzYMCZAK ${ }^{b}$ AND J. GonzALEZ ${ }^{c}$ \\ ${ }^{a}$ Department of Physics, Technical University of Radom \\ Malczewskiego 29, 26-600 Radom, Poland \\ ${ }^{b}$ Institute of Physics, Polish Academy of Sciences \\ Al. Lotników 32/46, 02-668 Warsaw, Poland \\ ${ }^{c}$ Departamento de Fisica de Materiales, Universidad del Pais Vasco \\ Paseo Manuel de Lardizabal, 3, 20009 San Sebastian, Spain
}

The magnetic anisotropy and magnetostriction in nanocrystalline grains in non-magnetic or magnetic matrix (nanoparticles or nanocrystalline materials) differ from their bulk properties. The interactions between atoms at the interface play the important role in these magnetic properties. In this paper the magnetic dipolar contribution to magnetostriction energy has been calculated for spherical crystal grains (of bcc and fcc symmetry) with interface layer of atoms possessing different magnetic moment. Total magnetoelastic energy of the sphere-interface system is a sum of magnetoelastic energy of the sphere, energy of the interactions between the sphere and the interface and also the energy of the interface. It was shown that magnetoelastic energy depends on the sphere size and interface thickness.

PACS numbers: $75.80 .+\mathrm{q}, 68.90 .+\mathrm{g}, 78.66 . \mathrm{Jg}$

The magnetic properties of materials such as granular systems, nanocrystalline alloys, thin films, and superlattices are determined by the properties of the interface between particular phases. The symmetry restrictions at surfaces and interfaces result in magnetic surface anisotropy (Néel, [1]) and, consequently, in surface magnetostriction. In this paper the magnetoelastic energy in crystal grains with different interfaces was calculated in the frame of dipolar magnetic interactions. The crystal grain was defined as a sphere cut out with bcc or fcc crystalline structure. The surrounding spherical layer of the interface has the same crystalline structure but different magnetic moment of atoms. The method of calculations of dipolar magnetoelastic energy is similar to that applied by Draaisma and de Jonge [2] to find the surface and volume anisotropy and by us to obtain surface magnetostriction in ultrathin ferromagnetic films [3] and nanoparticles [4]. 
A density of magnetostriction energy due to the dipolar interaction between magnetic moments $i$ and $j$ in sphere (oriented parallel to each other), can be expressed by $[3,4]$ :

$$
\frac{E_{\mathrm{ME}}^{(i, j)}}{V_{\mathrm{dip}}}=\frac{1}{2} \mu_{0} M^{2} b_{k l m n}^{(i, j)} \alpha_{k} \alpha_{l} \varepsilon_{m n}, \quad M=\frac{m_{\mathrm{sp}}}{V_{\mathrm{dip}}},
$$

where

$$
b_{k l m n}^{(i, j)}=-\frac{\partial D_{k l}^{(i, j)}}{\partial \varepsilon_{m n}}, \quad D_{k l}^{(i, j)}=\frac{V_{\mathrm{dip}}}{4 \pi}\left[-\frac{\delta_{k l}}{r_{i j}^{3}}+\frac{3\left(r_{i j}\right)_{k}\left(r_{i j}\right)_{l}}{r_{i j}^{5}}\right],
$$

$V_{\text {dip }}$ denotes the volume per one dipole, $m_{\mathrm{sp}}$ - the value of magnetic moment of atoms in sphere, $r_{i j}$ - relative position vector of the dipoles $i$ and $j, \alpha_{k}$ and $\alpha_{l}$ - direction cosines of magnetization $M$, and $\varepsilon_{m n}$ - deformation tensor. Second rank tensor $D_{k l}^{(i, j)}$ describing magnetic anisotropy energy and fourth rank tensor $b_{k l m n}^{(i, j)}$ describing the magnetoelastic energy are dimensionless and independent of lattice constant. In case of sphere with surrounding interface layer in which atoms possess different magnetic moment $m_{\text {if }}$, a total magnetostriction energy of the sphere-interface system is a superposition of three terms - the energy of the sphere itself, energy of the interactions between the sphere and interface, as well as the energy of the interface layer itself. The average, dimensionless magnetostriction energy tensor $b_{\mathrm{klmn}}^{(\mathrm{sp}+\mathrm{if})}$ for this system, as a function of relative magnetic moment $m_{\mathrm{r}}$, is given by the following expression:

$$
b_{k l m n}^{(\mathrm{sp}+\mathrm{if})}\left(m_{\mathrm{r}}\right)=\frac{N_{\mathrm{sp}} b_{k l m n}^{(\mathrm{sp})}+2 N_{\mathrm{sp}} b_{k l m n}^{(\mathrm{sp} / \mathrm{if})} m_{\mathrm{r}}+N_{\mathrm{if}} b_{k l m n}^{(\mathrm{if})} m_{\mathrm{r}}^{2}}{N_{\mathrm{sp}}+N_{\mathrm{if}}}
$$

in which

$$
\begin{aligned}
& b_{k l m n}^{(\mathrm{sp})}=\frac{1}{N_{\mathrm{sp}}} \sum_{i=1}^{N_{\mathrm{sp}}} \sum_{\substack{j=1 \\
j \neq i}}^{N_{\mathrm{sp}}} b_{k l m n}^{(i, j)}, \\
& b_{k l m n}^{(\mathrm{sp} / \mathrm{if})}=\frac{1}{N_{\mathrm{sp}}} \sum_{i=1}^{N_{\mathrm{sp}}} \sum_{j=N_{\mathrm{sp}}+1}^{N_{\mathrm{sp}}+N_{\mathrm{if}}} b_{k l m n}^{(i, j)}, \\
& b_{k l m n}^{(\mathrm{if})}=\frac{1}{N_{\mathrm{if}}} \sum_{i=N_{\mathrm{sp}}+1}^{N_{\mathrm{sp}}+N_{\mathrm{if}}} \sum_{\substack{j=N_{\mathrm{sp}}+1 \\
j \neq i}}^{N_{\mathrm{sp}}+N_{\mathrm{i} f}} b_{k l m n}^{(i, j)},
\end{aligned}
$$

and also $m_{\mathrm{r}}=m_{\mathrm{if}} / m_{\mathrm{sp}}$. The quantities given by (4) are the average tensors corresponding to three terms of magnetostriction energy, which have been specified above. $N_{\text {sp }}$ and $N_{\text {if }}$ are the numbers of atoms in sphere and interface layer.

The values of the first elements of the magnetoelastic energy tensors has been calculated numerically for several sphere radii $\left(R_{\mathrm{sp}}=4, \ldots, 8 a_{0}\right.$, where $a_{0}$ is a lattice constant) and for different thickness of interface layer $\left(h_{\mathrm{if}}=1, \ldots, 4 a_{0}\right)$. They are presented in Fig. 1 as a function of $N_{\mathrm{s}} / N_{\mathrm{sp}}$, where $N_{\mathrm{s}}$ is a number of atoms in surface layer of sphere. For example in bcc sphere of radius $R_{\mathrm{sp}}=4 a_{0}$ one has 
$N_{\mathrm{sp}}=537$ and $N_{\mathrm{s}}=278$ whereas for $R_{\mathrm{sp}}=8 \mathrm{a}_{0}$ these numbers are equal to 4285 and 1394 , respectively. The average magnetostriction energy of spherical crystallite $\left(b_{1111}^{(\mathrm{sp})}\right)$ reveals almost linear dependence as well as sphere-interface interaction term $\left(b_{1111}^{\text {(sp/if) }}\right)$ which is approximately independent of the interface thickness. The magnetoelastic energy of the interface layer $\left(b_{1111}^{(\mathrm{if})}\right)$ depends weakly on sphere size, but it is very sensitive on the interface thickness. The results are qualitatively similar for bcc and fcc crystalline structure.
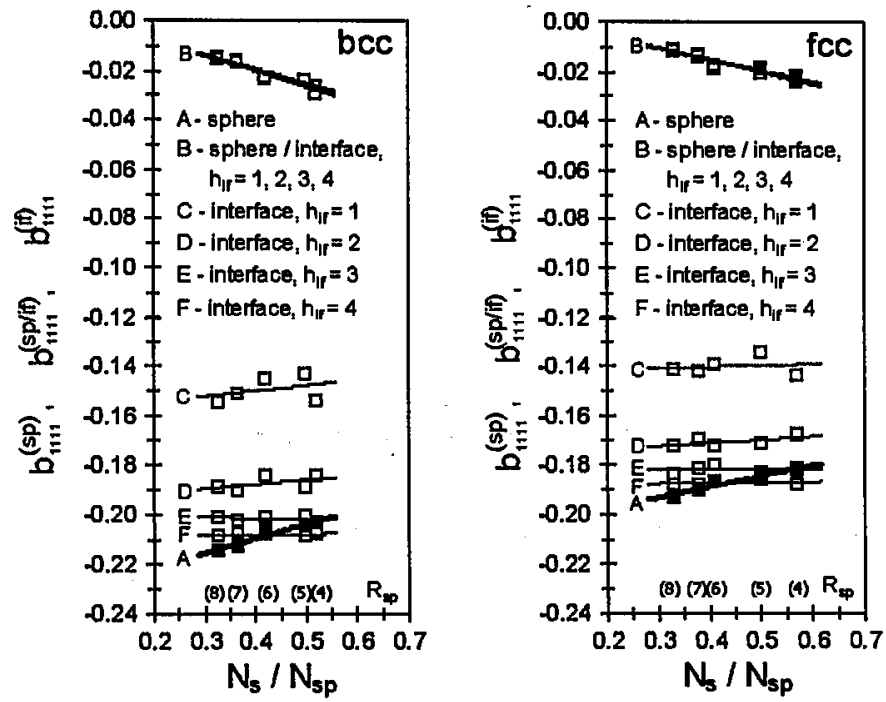

Fig. 1. The average, dimensionless tensor elements describing the magnetostriction energy of the sphere $b_{1111}^{(\mathrm{sp})}$, sphere/interface interaction $b_{1111}^{\text {(sp/if) }}$ and the interface $b_{1111}^{(\text {(if })}$ for the bcc and fcc crystalline structure. The calculations were done for various sphere radii $R_{\text {sp }}$ (i.e. different ratio of the number of surface atoms in sphere $N_{s}$ to the total number of atoms in the sphere $N_{\mathrm{sp}}$ ) and different thickness of interface layer $h_{\mathrm{if}}$. To the numerical results the "trend lines" were fitted.

Using Eq. (3), the average magnetostriction energy tensor elements $b_{1111}^{\text {(sp }+ \text { if) }}$ for the sphere-interface system have been plotted in Fig. 2 as a function of the ratio $m_{\mathrm{r}}=m_{\mathrm{if}} / m_{\mathrm{sp}}$ of magnetic moment of atoms in the interface to its value for atoms in the sphere. This dependence is described by cubic polynomial of $m_{\mathrm{r}}$. It is strong for the small spheres and thick interfaces $\left(R_{\mathrm{sp}}=4 a_{0}, h_{\mathrm{if}}=4 a_{0}\right)$, because the number of atoms in the interface is significantly bigger than number of atoms in the sphere (e.g. for bcc structure $N_{\mathrm{sp}}=537$ and $N_{\text {if }}=3748$ ). On the contrary, the dependence is more weak for large spheres and thin interfaces $\left(R_{\text {sp }}=8 a_{0}\right.$, $\left.h_{\mathrm{if}}=1 a_{0}\right)$, since the number of atoms in sphere is predominating relatively to the number of atoms in the interface (for bcc structure $N_{\mathrm{sp}}=4285$ and $N_{\text {if }}=1898$ ). The negative values of $m_{\mathrm{r}}$ correspond to the case of the antiferromagnetic coupling between spins in the sphere and in the interface. Symmetry relations enable to express other elements of magnetostriction energy tensor by $b_{111}^{\text {(sp+if) }}$ element. 

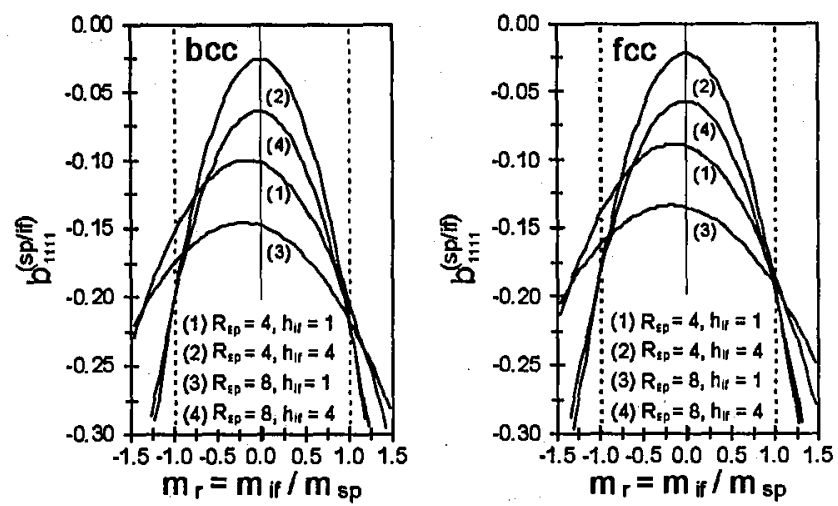

Fig. 2. The dependence of a verage magnetostriction energy tensor element $b_{1111}^{(\mathrm{sp}+\mathrm{if})}$ for the bcc and fcc sphere-interface system on the ratio of magnetic moment of atoms in the interface to the magnetic moment of atoms in the sphere $m_{\mathrm{r}}=m_{\mathrm{if}} / m_{\mathrm{sp}}$.

In conclusion, it should be stressed that interface layer gives an important contribution to the total magnetostriction of crystallite-interface system. A different value of magnetic moment of atoms in the interface modifies strongly the a verage magnetoelastic energy in case of small crystallites. In general, the dependence of magnetostriction on crystallite radius is specially visible for the small radii. Our results convince of the existence of surface magnetostriction in nanostructured systems. It is additionally supported by the fact that interface layer influences magnetostriction energy first of all in the last (external) layer of crystallite, which has been shown in [5]. The magnetostriction model, based on magnetic dipole-dipole interactions and presented in this paper, could be useful for describing the surface and interface effects of magnetostriction in nanoparticles and nanocrystalline materials.

This work was supported in part by the Committee for Scientific Research.

\section{References}

[1] L. Néel, J. Phys. Radium 15, 225 (1954).

[2] H.J.G. Draaisma, W.J.M. de Jonge, J. Appl. Phys. 64, 3610 (1988).

[3] T. Szumiata, H. Szymczak, R. Żuberek, IEEE Trans. Magn. 29, 3132 (1993).

[4] R. Żuberek, T. Szumiata, A. Ślawska-Waniewska, H. Szymczak, Non-Crystalline and Nanoscale Materials, Eds. R. Rivas, M.A. Lopez-Quintela, World Scientific, Singapore 1998, p. 505.

[5] T. Szumiata, R. Żuberek, J. Gonzalez, A. Ślawska-Waniewska, H. Szymczak, J. Magn. Magn. Mater. 203, 262 (1999). 